\title{
Uniform surface modification of polyethylene terephthalate (PET) by atmospheric pressure plasma jet with a horn-like nozzle
}

\author{
Taiana She Mir Mui ${ }^{a}$,* Rogério Pinto Mota ${ }^{a}$, Antje Quade ${ }^{b}$, Luis Rogério de Oliveira Hein ${ }^{a}$, \\ Konstantin Georgiev Kostov ${ }^{\mathrm{a}}$
}

a São Paulo State University - Unesp, Faculty of Engineering - FEG, 12516-410 Guaratinguetá, SP, Brazil

${ }^{\mathrm{b}}$ Leibniz Institute for Plasma Science and Technology - INP-Greifswald, D-17489 Greifswald, Germany

\section{A R T I C L E I N F O}

\section{Keywords:}

Atmospheric pressure plasma jet

Horn-like nozzle

Surface modification

PET

\begin{abstract}
A B S T R A C T
This work reports on surface modification of polyethylene terephthalate (PET) polymer by an atmospheric pressure plasma jet (APPJ) operated with argon. A distinguishable feature of this device is that it terminates with a conical horn-like nozzle. Three different nozzles diameters were employed with the purpose to obtain uniform surface modification over large area. Treatments in small 3D objects that fit inside the conical horn were also conducted. In this study, water contact angle (WCA) measurements and X-ray photoelectron spectroscopy (XPS) were performed to assess the samples wettability and the surface elemental composition, as well as, their radial distribution. Plasma-induced changes on the polymer surface morphology were evaluated by Atomic Force Microscopy (AFM). Electrical characterization of the plasma and investigation of the effect of the gas flow rate on the discharge power were carried out. After the plasma treatment PET surface became more hydrophilic over the entire area covered by the nozzle. This effect is caused by the incorporation of oxygen containing polar groups on the surface. It was also observed that depending on process parameters, the plasma treatment can extend even outside the area of the conical horn. The degree of surface modification depends on plasma dose while the treatment uniformity is determined mostly by the distance to the sample. Overall, a quite uniform surface modification was obtained over the entire area covered by the jet nozzle. Thus, the results suggest that by simply changing the jet geometry and choosing the right treatment parameters one can achieve a uniform treatment over an area whose size is determined by the horn diameter.
\end{abstract}

\section{Introduction}

In the last decade, cold atmospheric plasmas (CAPs) have been extensively investigated because their operation does not require vacuum vessels and pumping systems thus allowing compact and low-cost plasma sources that can be easily employ in material processing and biomedicine as well as in continuous in-line production [1]. To generate and maintain non-thermal plasmas at atmospheric pressure several methods, such as, asymmetric electrode configurations, dielectric barriers, high gas flows and submillimeter plasma dimensions have been used. Among these approaches the dielectric barrier discharge (DBD) is definitely the most preferred method for surface treatment, especially when modification over large area (like in textile industry) is required [2]. However, the plasmas generated in atmospheric DBDs are confined into small gaps, thus making impossible the treatment of complex-shaped 3D samples. To overcome this problem in the late 90s were introduced so-called cold atmospheric pressure plasma jets (APPJs) [3]. In these devices electric discharge is usually ignited in a noble gas that flows through a thin dielectric tube and the resulting plasma is ejected into the surrounding environment (usually air) [4]. Depending on the jet operating conditions the gas temperature can be kept close to the room temperature [5]. However energetic plasma species (electrons, photons and metastables) interact with air molecules producing reactive oxygen (ROS) and nitrogen (RNS) species that can modify and decontaminate surfaces [5,6]. Since APPJs can generate up to several $\mathrm{cm}$-long plasma plumes they can be adapted to treat irregular $3 \mathrm{D}$ objects and also internal surface of narrow tubes or cavities [7]. In most excitation schemes and working gases the plasma plume diameter is slightly wider than the inner diameter of the employed dielectric tube $[3,5,6]$. Therefore for localized treatments, as in dentistry or microelectronics, usually ultrafine capillaries with diameter of $0.1-1.0 \mathrm{~mm}$ are used $[8,9]$. The long-living active species generated in the plasma

\footnotetext{
* Corresponding author at: Department of Physics and Chemistry, Universidade Estadual Paulista Júlio de Mesquita Filho (UNESP), Faculdade de Engenharia de Guaratinguetá, Av. Ariberto Perreira da Cunha 333, Pedregulho, 12516-410 Guaratinguetá, SP, Brazil.

E-mail address: taiana.mui@unesp.br (T.S.M. Mui).
} 


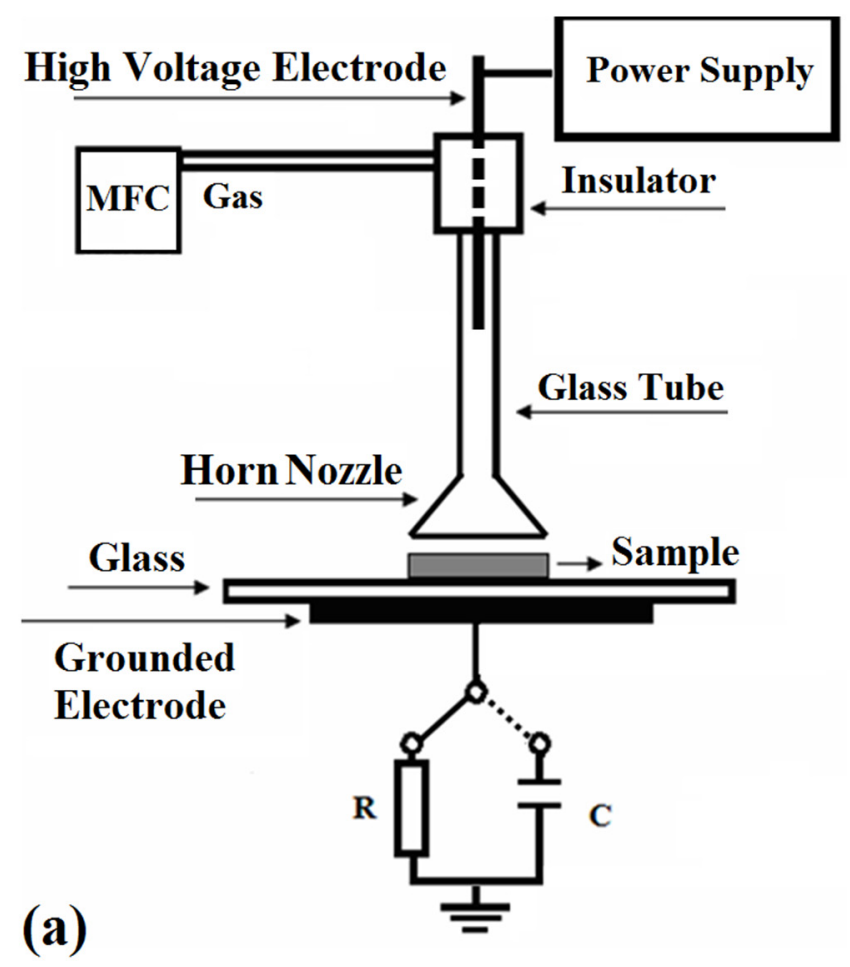

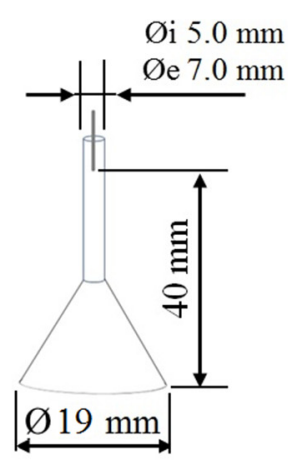

(b)

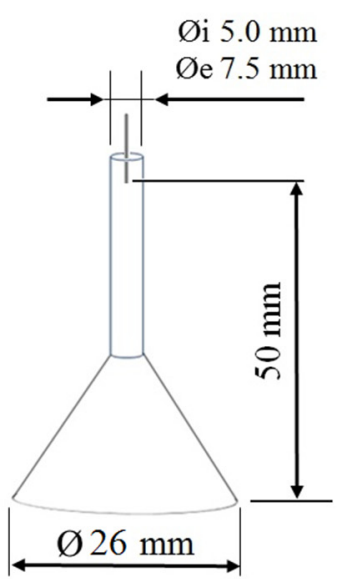

(c)

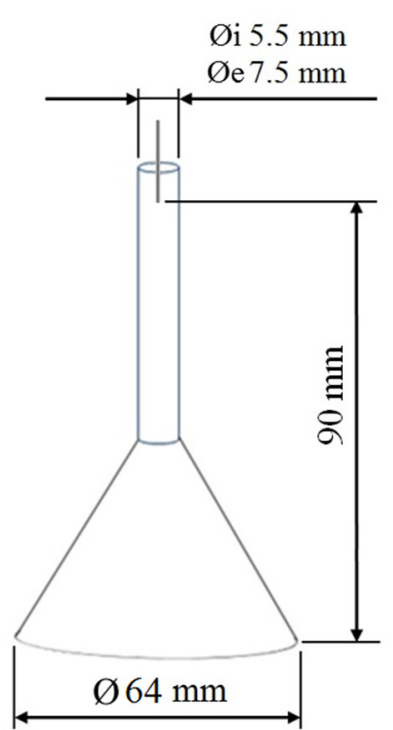

(d)

Fig. 1. (a) Experimental setup. Plasma jets dimensions: (b) small jet; (c) medium jet and (d) big jet.

plume diffuse radially outward forming a reactive zone, which is two to three times larger than the plasma column itself. Inside this circular zone, whose size in the most experiments ranges from few $\mathrm{mm}^{2}$ to about few $\mathrm{cm}^{2}$ [10-12], the material surface properties can be altered [11]. Surface modification of different polymers by atmospheric plasmas has been extensively investigated in the literature [11-15] and although the exact mechanisms is not yet fully understood, the oxygen incorporation on the surface and the polymer chain crosslinking were appointed as the main reasons behind the increase of material surface energy $[12,13]$. The latter results in enhanced wettability and adhesion properties that are very much desirable for coating, printing, painting and dying. All these applications require plasma sources that can generate plasma over large area $[14,15]$. These devices are typically built in DBD geometry and use moving stages or rotating drums, components that can be readily implemented only for treatment of thin films. However, for bulky and irregular samples the plasma jet rather than the DBD configuration should be used resulting in a surface modification, which is restricted over small area (typically few $\mathrm{cm}^{2}$ ), and is also not uniform [16]. To remedy this disadvantage of conventional APPJs different approaches have been attempted. First of all, simply increasing the diameter of the dielectric tube is not a plausible solution because it leads to excessively high gas flow rates and also increasingly higher electric fields are required for plasma ignition. Therefore for large area applications authors usually design arrays of closely packed plasma jets 


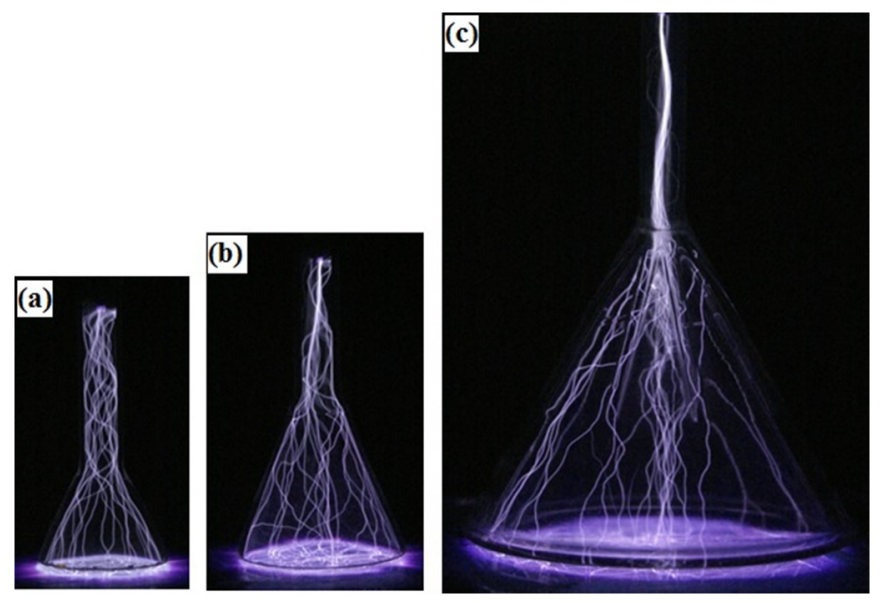

Fig. 2. Photos of: (a) small; (b) medium and (c) big plasma jet in operation. Distance to the target $1 \mathrm{~mm}$. Parameters: small $\left(20 \mathrm{kV}_{\mathrm{p}-\mathrm{p}}, 3.0 \mathrm{~ms}, 2.0 \mathrm{slm}\right)$, medium (20 kV $\left.\mathrm{k}_{\mathrm{p}-\mathrm{p}}, 2.2 \mathrm{~ms}, 2.0 \mathrm{slm}\right)$, big $\left(16 \mathrm{kV}_{\mathrm{p}-\mathrm{p}}, 1.7 \mathrm{~ms}, 2.0 \mathrm{slm}\right)$.

operated in parallel [17]. The jets are combined into honeycomb [18] or linear [19] structures and share the same gas feed and power supply. However, as reported by some authors [20] the individual jets interact with each other (hydro-dynamically by their gas flows and electrically by their electric charges) thus making hard to ignite all plasma jets in a uniform manner. Also, jets at the array edge commonly exhibit different properties (like jet length, plume deviation from the normal etc.) from jets in the central area so that the treatment uniformity is compromised. Another method to obtain a large area plasma plume at atmospheric pressure relies on cooperative merging of two or more individual jets into a wide plasma column (up to 2-cm-diam) as reported in [21,22]. However, the jets merging works properly only within a limited range of gas flows and applied voltages and with $\mathrm{He}$ as working gas, which is not feasible for surface processing applications. Finally, the dielectric tube size and termination, as well as, the electrodes type, shape and configuration can significantly influence the plasma jet and its shape and length [23-25]. For instance the authors in [25] obtained a 2-cmdiam plasma jet near atmospheric pressure by using three (a powered, a grounded and a floated) electrodes. Using dielectric tubes with different exit geometry, like a tapered or horn-like nozzle as reported in [26], changes the gas flow dynamics, which affects the length and size of the produced plasma jet. Yet, there is no comprehensive study about the effect of jet exit geometry on the size and the uniformity of plasmamodified area.

PET is the most common thermoplastic polymer in the world. It has excellent properties such as chemical resistance, easy recycling, resistance to corrosion, high flexural modulus and superior dimensional stability [16]. Due to its unique qualities (strength, versatility, and recyclability) PET has been employed in a wide range of application from food, beverages and cosmetics package to textiles manufacture. However, polymers in general have a major drawback due to their low surface free energy, leading to poor wettability and adhesion of paintings, glues or printings $[14,15]$. Therefore, surface treatments are often required for polymers. As has been said before, non-thermal plasmas is one of the environmental- friendly techniques that have been applied to surface modification of polymers, with the purpose to enhance their surface wettability and adhesion properties [11-15].

In this work we deal with surface modification of PET polymer by an atmospheric pressure plasma jet operated with Ar. The device basically consists of an exchangeable Pyrex glass funnel, which terminates with a conical nozzle. Depending on jet operating conditions, such as jetsample distance, funnel exit diameter and gas flow rate, uniform surface modification over the entire area under the horn can be achieved. Therefore, a uniform surface modification over relatively large area $\left(\sim 30 \mathrm{~cm}^{2}\right)$ can be obtained by simply increasing the funnel diameter and scaling up the jet operation parameters. Moreover, it was shown that polymer samples that were placed vertically in the horn were also treated in a uniform matter. So we propose a simple method that can be successfully implemented to treated $3 \mathrm{D}$ objects as for example in the agriculture for plasma treatment of seeds.

\section{Material and methods}

\subsection{Plasma jet with horn-like nozzle}

Fig. 1(a) depicts a schematic drawing of the plasma jet configuration employed in this work. It consists of a pin electrode $(1 \mathrm{~mm}$ thick $\mathrm{Ni}$ $\mathrm{Cr}$ wire) centered inside a Pyrex glass conical funnel. The device was installed vertically with the funnel exit pointing downward. Polymers samples with diameter larger than the horn diameter were fixed on a 6mm-thick glass table with a grounded metal electrode $(\varnothing 155 \mathrm{~mm}$; thickness of $1.5 \mathrm{~mm}$ ) beneath it. Three plasma jets with very similar tube diameters but rather different funnel's exit dimensions were studied in this work. The jets were designated accordingly to their funnel's inner diameter as a small jet ( $\varnothing 19 \mathrm{~mm}$ ), a medium jet $(\varnothing 26 \mathrm{~mm})$ and a big jet ( $\varnothing 64 \mathrm{~mm}$ ) and Fig. 1(b-d) exhibit jets drawings with their exact dimensions. The size, length and thickness of the glass tubes are slightly different because we used some commercially available glass funnels.

Argon gas was admitted into the system through a Teflon holder and gas flow rate was adjusted by a mass-flow controller Horiba model STEC N100. Plasma was excited by an AC power supply (Minipuls 4, GBS Elektronik, Dresden, Germany), which was operated in burst mode. The generated waveform was an amplitude-modulated sinewave, i.e. a constant period of high-voltage oscillations is followed by a voltage off period. The number of high-voltage cycles $(\mathrm{N}=12)$ and the signal frequency $(25 \mathrm{kHz})$ were kept constant, while other parameters like burst repetition period and voltage magnitude were adjusted. For given operation conditions, the plasma in all jets was generated in the form of randomly distributed filaments that emerged from the powered pin electrode, propagated along the inner wall of the glass funnel until hit the target. Photos of the three plasma jets in operation are shown in Fig. 2 for distance to the target of $1 \mathrm{~mm}$.

\subsection{Power calculation}

The transferred charge and the discharge current waveform were obtained by measuring the voltage drop across a serial capacitor of $10 \mathrm{nF}$ or a serial resistor of $47 \Omega$, respectively. The high-voltage signal was measured by a voltage divider $(1 \times 2000)$ installed directly in the power generator. The signals were monitored on a digital oscilloscope (Tektronix TDS 3032C, $300 \mathrm{MHz}$ ).

To determine the plasma jet mean power we used the Q-V Lissajous method described before [27]. It was adapted for amplitude-modulated voltage signals as reported in our previously work [28]. Basically, the total energy of a high-voltage oscillation group $\left(E_{T}\right)$ was obtained from the area of the Lissajous figure $(\mathrm{QxV})$ and then, it was divided by the burst repetition period $\left(T_{r}\right)$, as shown in Eq. (1):

$P=\frac{E_{T}}{T_{r}}$

It is known from the literature that distance to the target as well as the target dielectric properties affect the plasma jet operation and more specifically its power [29]. Usually at very small distances the plasma jet power tends to increase abruptly, which makes the comparison between different treatments very difficult. Therefore using amplitudemodulated voltage is an advantage since it allows better control of the jet power. For instance, at shorter distances, when the plasma jet energy per voltage cycle increases, one can select a longer burst repetition period in this way maintaining the same mean power. Using this method one can maintain fixed some parameters, such as, voltage amplitude, plasma power, power density or plasma dose, which is very 
Table 1

Plasma jets parameters.

\begin{tabular}{|c|c|c|c|c|c|c|c|}
\hline Jet & Nozzle internal diameter (mm) & Voltage $\left(\mathrm{kV}_{\mathrm{p}-\mathrm{p}}\right)$ & Period $\mathrm{T}_{\mathrm{r}}(\mathrm{ms})$ & Power (W) & Power per unit area $\left(\mathrm{W} / \mathrm{cm}^{2}\right)$ & Treatment time (s) & Energy dose $\left(\mathrm{J} / \mathrm{cm}^{2}\right)$ \\
\hline Small & 19 & 20.0 & 3.0 & 3.4 & 1.20 & 20 & 24.0 \\
\hline Medium & 26 & 20.0 & 2.2 & 6.1 & 1.15 & 20 & 23.0 \\
\hline Big & 64 & 16.0 & 1.7 & 10.5 & 0.33 & 70 & 23.1 \\
\hline
\end{tabular}

useful when comparing treatment results obtained by plasma jets with different configurations.

The process parameters used in this study are listed in the Table 1. As can be seen the plasma jet power scales with the funnel exit size. Therefore to be able to compare surface modification efficiency of different jets we selected two key treatment parameters: the power per unit area and the plasma dose delivered to the target. The power per unit area is calculated dividing the jet power by the area of the conical horn. The small and the medium jet parameters were chosen in a way to get the same power density $\left(\sim 1.20 \mathrm{~W} / \mathrm{cm}^{2}\right)$. However, in the case of big plasma jet (whose size is more than twice the size of the medium jet) our power supply was not able to deliver sufficient power to obtain the same power per unit area. Therefore, in this case to achieve the same energy dose as for the other two jets we set a longer treatment time. So in this way the three different jets delivered to the target about the same energy dose $\left(\sim 23 \mathrm{~J} / \mathrm{cm}^{2}\right)$ and thus their surface modification effect can be compared. In the case of the big plasma jet however longer treatment time can be considered as a disadvantage. On the other hand this jet has the lowest power per unit area, which makes it suitable for treatment of thermosensitive and biological materials.

To optimize plasma treatment parameters in a separated study we investigated the effect of nozzle to sample distance on the PET surface wettability. In addition, the dependence of jet mean power on gas flow rate for the three different funnel exits was also investigated.

\subsection{PET substrates}

In the last decade surface modification of materials by atmospheric plasma has been extensively studied and in the literature can be found many papers reporting on treatment of different engineering and biocompatible polymers by APPJs. Details about this subject can be found in a recent review paper [6]. However, in this study we focus on increasing plasma-treated area and improving process uniformity rather than investigating the mechanisms behind plasma surface interaction, which has been addressed in previous works [14,15]. For that purpose we chose Polyethylene Terephthalate (PET) as a standard engineering polymer, which is cheap, easy to handle and also because lots of data about PET plasma proceeding is available for comparison [16].

All plasma treatments were performed on 0.3-mm-thick PET samples cut from commercial plastic bottles. For each jet samples with different sizes were used: e. g. $40 \times 90 \mathrm{~mm}$ (small and medium jets) and $80 \times 90 \mathrm{~mm}$ (big jet). Also, in order to simulate treatments of bulky targets we introduced so-called vertical treatment. It was performed by the big jet using the parameters as in Table 1. Differently from the previous treatments the target - a 1-mm-thick glass slide holding two PET samples $35 \times 25 \mathrm{~mm}$ (one on each slide side) was placed standing upward inside the conical horn, as shown in Fig. 3.

Before plasma treatment, all samples were first ultrasonically cleaned in distilled water for $15 \mathrm{~min}$ and after that in isopropyl alcohol for $10 \mathrm{~min}$ to remove organic contaminants and finally left to dry at room temperature for $1 \mathrm{~h}$. The PET surface temperature was monitored by a thermocouple, which was put in contact with the treated surface immediately after the treatment. For all treatment conditions the measured values did not exceed $50^{\circ} \mathrm{C}$.

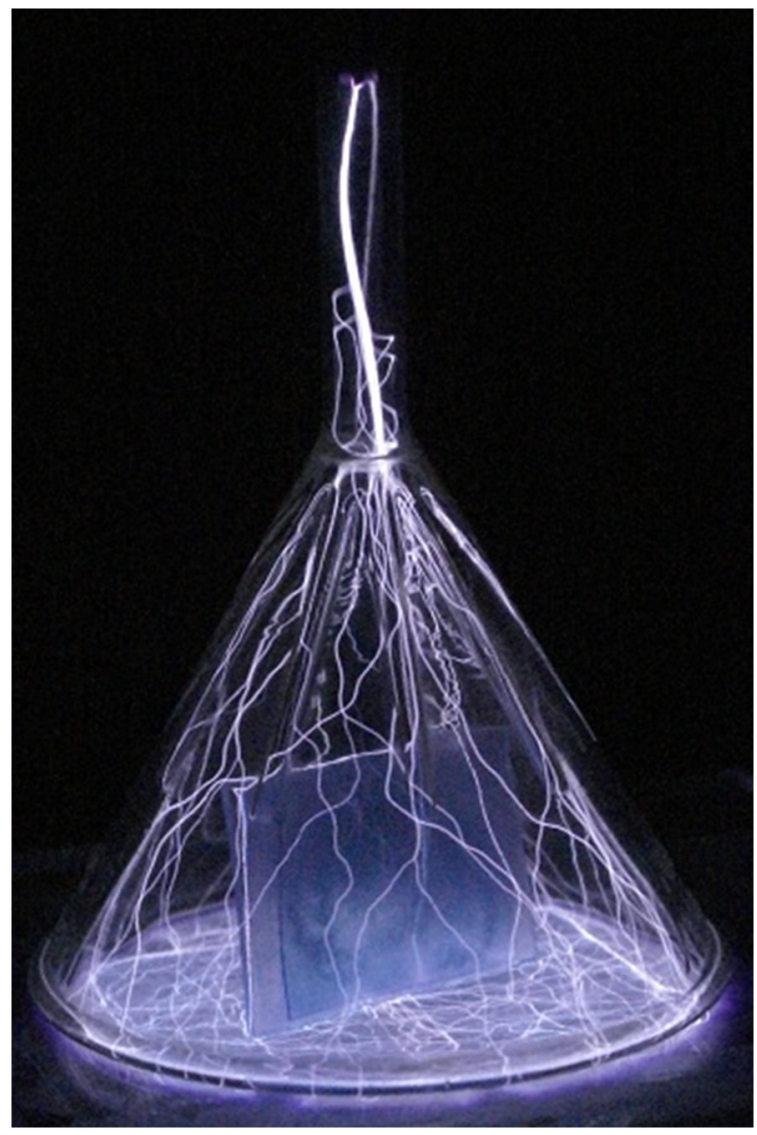

Fig. 3. Treatment of a vertical target with the big jet. Parameters: $\left(16 \mathrm{kV}_{\mathrm{p}-\mathrm{p}}\right.$, $1.7 \mathrm{~ms}, 2 \mathrm{slm})$.

\subsection{Water contact angle (WCA) measurements}

Water contact angle measurements were obtained using the sessile drop method on a Rame-Hart goniometer (300 F1) with automated drop dispenser. Deionized water was used as a test liquid and the volume of each drop was set as $1.0 \mu \mathrm{l}$. For each sample 21 droplets were deposited equidistantly on the PET surface in order to determine the treatment area and the radial distribution of the WCA. To verify the reproducibility all experiments were done in triplicate and the mean value of WCA, as well as the standard deviation were calculated. The WCA measurements were performed within 10 min interval after the plasma treatment.

\subsection{XPS analysis}

Immediately after the plasma exposure three PET samples (S2, S3 and S4), treated by the jet with the small, medium and big exit horn, respectively, were cut along their central line (following the samples longer side) into 90-mm-long, 5-mm-wide stripes. Only for the PET sample (S5) that was placed vertically inside the plasma jet with the big conical horn, the stripe length was $35 \mathrm{~mm}$. Those stripes together with a pristine sample (S1) were subjected to XPS analyses in INP-Greifswald, Germany within 1 week after the treatment. The measurements were 
performed with a X-ray photoelectron spectrometer Axis Ultra, Kratos, Manchester, UK, equipped with a monochromatic Al Ka X-ray source $(1486.6 \mathrm{eV})$. The Axis Ultra is a high performance scanning XPS device with energy resolution $1.0 \mathrm{eV}$ on polymers and local resolution better than $15 \mu \mathrm{m}$. The XPS analysis is restricted to depths of some nms. The detection limit is between $0.1 \%$ and $1 \%$ depending on local resolution and chemical element which is under investigation. The hydrocarbon component of the C1s peak $(285.0 \mathrm{eV})$ was used to calibrate the energy scale. The results were processed with CasaXPS 2.3.15 and Origin7G software.

Three kinds of XPS measurements were performed:

a) a wide scan (1 measurement per sample) with $150 \mathrm{~W}$ of X-ray radiation $(15 \mathrm{kV}, 10 \mathrm{~mA})$, spot size $250 \mu \mathrm{m}$ and pass energy of $80 \mathrm{eV}$.

b) line scans ( $5 \mathrm{~mm}$ step for samples $\mathrm{S} 2, \mathrm{~S} 3, \mathrm{~S} 4$; and $3 \mathrm{~mm}$ step for S5) of O1s, C1s, N1 s, Si 2p, Cl 2p-peaks. Scan parameters: $150 \mathrm{~W}$ of Xray radiation $(15 \mathrm{kV}, 10 \mathrm{~mA})$, spot size $250 \mu \mathrm{m}$ and pass energy of $80 \mathrm{eV}$.

c) Highly resolved C1s peak line scans $(10 \mathrm{~mm}$ for S2, S3, S4; and $6 \mathrm{~mm}$ for S5). Parameters: $225 \mathrm{~W}$ of X-ray radiation $(15 \mathrm{kV}, 15 \mathrm{~mA})$, spot size $250 \mu \mathrm{m}$, pass energy $10 \mathrm{eV}$.

\subsection{Atomic Force Microscopy}

Atomic Force Microscopy (AFM) was employed to assess the changes in the polymer surface morphology. The AFM analysis was performed by a Shimadzu (SPM-9600) Scanning Probe Microscope. It was operated in tapping mode with a scanning rate of $0.5 \mathrm{~Hz}$ and an etched silicon probe Tap 190-G $(\mathrm{k}=48 \mathrm{~N} / \mathrm{m})$ was used for all scanning. From the topographic profiles on $2 \times 2 \mu \mathrm{m}^{2}$ area, the root mean square (rms) roughness of the surface was evaluated using the freeware software Gwyddion 2.5.

\section{Results and discussion}

\subsection{Electric characterization}

The three plasma jets with different exit nozzles exhibited very similar Lissajous figures and waveforms of the discharge current. Therefore, here only one set of measurements, the one for the small plasma jet, is illustrated in Fig. 4. Fig. 4(a) displays a characteristic voltage waveform showing the repetition period between two highvoltage voltage oscillations groups, also known as bursts [29]. In this work the number of high-voltage oscillation inside a burst was fixed to 12 while the repetition period was varied from $1.0 \mathrm{~ms}$ to $3.0 \mathrm{~ms}$ in order to achieve the desired discharge power. In the Fig. 4(b) are shown the typical current and voltage waveforms taken in a timeframe of $100 \mu \mathrm{s}$. The current signal exhibits several short current peaks (with more peaks in the positive half-period than in the negative one) superimposed to a capacitive current. In the Fig. 4(c) is presented the Lissajous figure obtained during one burst. It consists of number of enclosed loops, one for each cycle of high-voltage oscillation.

\subsection{Nozzle to sample distance}

The effect of nozzle to sample distance was investigated for the small jet. The other two jets exhibited very similar behavior and thus are not presented here. Treatment time was set to 20 s and the mean power and the gas flow rate were $3.4 \mathrm{~W}$ and $2.0 \mathrm{slm}$, respectively. Fig. 5(a-c) shows the radial distribution of WCA on PET surface for three distances of 1,2 and $3 \mathrm{~mm}$. As can be seen for all cases the WCA radial distribution has approximately trapezoidal shape with a flat bottom region approximately equal to the nozzle's size and a characteristic width (FWHM) about twice the horn diameter. Outside the horn plasma modification effect falls down linearly until the WCA reaches the value of pristine polymer. Incrementing distance to the target the minimal value of WCA and its distribution are roughly preserved (tough for $3 \mathrm{~mm}$ distance the plateau size is reduced) however the uncertainty of WCA measurements increases significantly. It can be concluded that for smaller distance the treatment results are more reliable and reproducible. Therefore, the nozzle to sample distance was kept constant as $1 \mathrm{~mm}$ in all further experiments with the three different jets.

\subsection{Effect of the gas flow on power}

Usually the operation of conventional plasma jets with straight tube exit is very much affected by the gas flow. The discharge power, plasma plume length and plasma spreading over a target depend upon the gas flow rate [30]. However, in a configuration with large conical nozzle the effect of gas flow turns out to be much less important. Strictly speaking in this case there is no conventional plasma jet although at very small horn-to-sample distances $(1-2 \mathrm{~mm})$ and high gas flows one can observe a plasma plume spreading radially outward from the horn edge. The effect of the gas flow rate on the discharge power was investigated and the results are shown in Fig. 6 . The idea behind this experiment was to determine for each plasma jet which is the most appropriate gas flow rate for performing surface modification experiments.

Below $1.2 \mathrm{slm}$ of Ar flow the operation of all jets was unstable since the filaments could not reach the target. In the whole range from $1.2 \mathrm{slm}$ to $3.0 \mathrm{slm}$ we have a relatively stable jets operation with discharge power that only slightly increases (practically within the measurements uncertainty) with the flow rate. Therefore, the gas flow was fixed to $2.0 \mathrm{slm}$ for all jets, since the plasma generation was more stable at this condition.

\subsection{Water contact angle}

\subsubsection{Horizontal PET samples}

Fig. 7 illustrates the water contact angle distribution along the samples longer dimension $(90 \mathrm{~mm})$ for all plasma jets. The nozzle geometrical center was placed above the zero point of the axis. The energy dose of the treatment is given in Table 1 and the distance to the sample was $1 \mathrm{~mm}$ in all cases.

As can be seen in the Fig. 7 the three plasma jets induced a considerable increase of sample wettability (minimum WCA of the treated sample $\sim 25^{\circ}$ ) when comparing to the untreated PET (WCA of $80^{\circ}$ ). Those WCA values are in a close agreement with previous results about plasma treatment of PET reported in literature [31-33]. When approximately equal energy dose is delivered to the samples the minimal value of WCA obtained for all plasma jets is the same, but the width of WCA radial distribution scales with the horn dimension. The plateau of WCA distribution is approximately equal to the horn diameter so that the treated area with uniform wettability increases from 3.0, 12.5 and $30.0 \mathrm{~cm}^{2}$ for the three jets, respectively. These results suggest that by changing the plasma jet nozzle geometry it is possible to uniformly modified area up to tens of $\mathrm{cm}^{2}$ with feasible operating conditions (Ar as working gas at low flow rate). As a matter of fact uniform surface modification over large area using a plasma jet is still a considerable challenge. Some authors [16] have also attempted a novel plasma jet design in order to improve the treatment uniformity of PET polymer. Even though they report very similar reduction of the WCA the treated area was much smaller the treatment uniformity was much worse compare to the ones obtained in this study.

\subsubsection{Treatment of vertical PET samples}

Another problem for technological application of atmospheric pressure plasma jets is the treatment of 3D samples without using sophisticated displacement systems. The plasma jet with big nozzle is large enough so that some small bulky objects, like seeds or small fruits can be fitted inside and treated by plasma. However, it is unclear whether the surface modification of such objects will be uniform or not. 


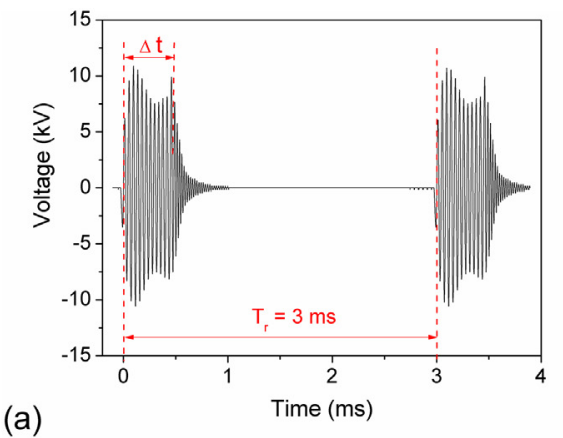

(a)
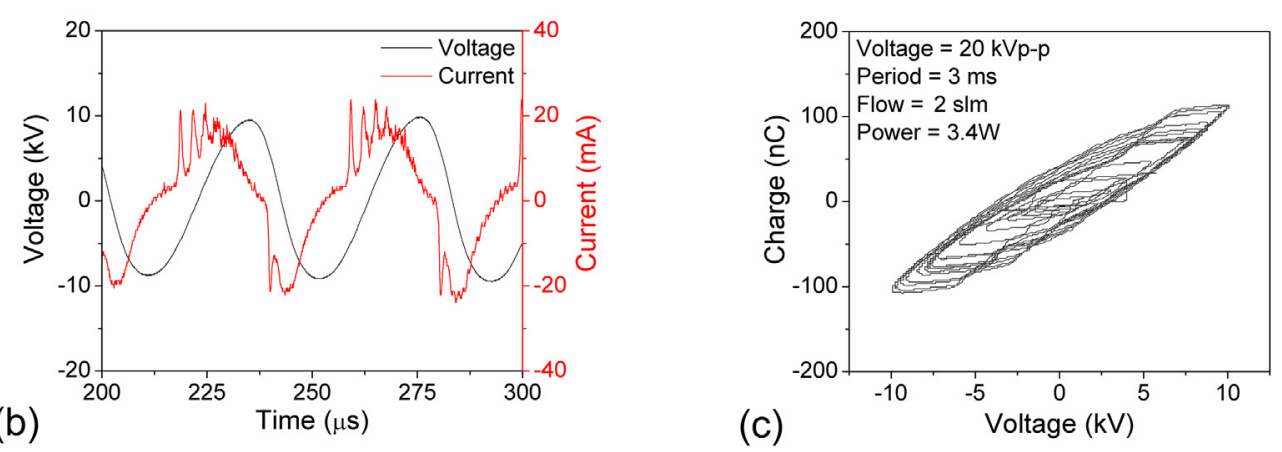

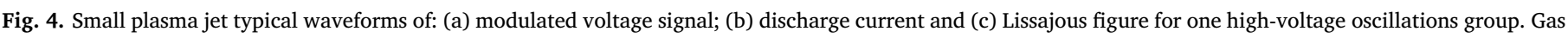
flow rate and the distance to the target were $2.0 \mathrm{slm}$ and $1 \mathrm{~mm}$, respectively.

In attempt to respond this question we used PET samples that were placed vertically in the jet conical horn as shown in the Fig. 3. After plasma treatment performed at the same conditions as for horizontal substrates, the WCA was measured on both sides of the target and the results are plotted in Fig. 8 as a function of the sample height. As can be

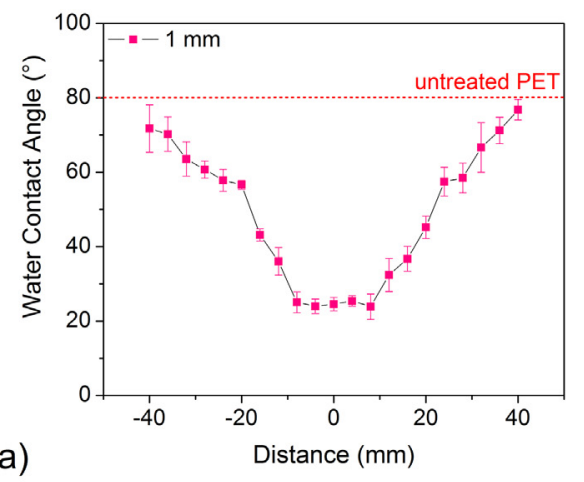

seen the treatment resulted in a quit uniform WCA distribution on both sample sides. Although the minimum value of WCA $\left(\sim 35^{\circ}\right)$ is bigger than the one for horizontal samples $\left(\sim 25^{\circ}\right)$ one can still speculate real 3D samples could be indeed treated by this method and in a relatively uniform way.

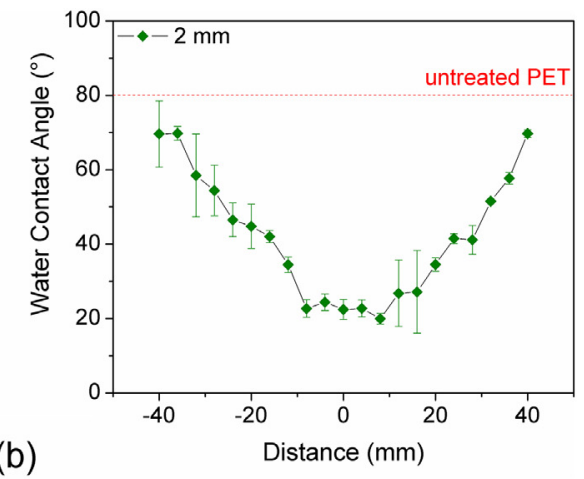

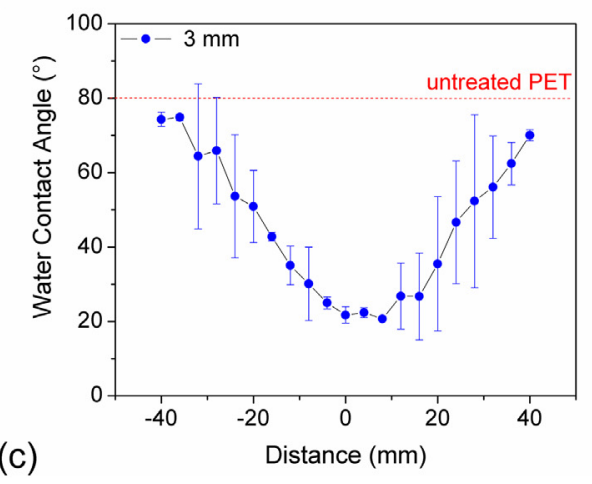

Fig. 5. WCA vs nozzle to sample distance (a) $1 \mathrm{~mm}$; (b) $2 \mathrm{~mm}$ and (c) $3 \mathrm{~mm}$. the samples were treated by the small plasma jet operated with Ar flow of 2.0 slm and power of $3.4 \mathrm{~W}$. 


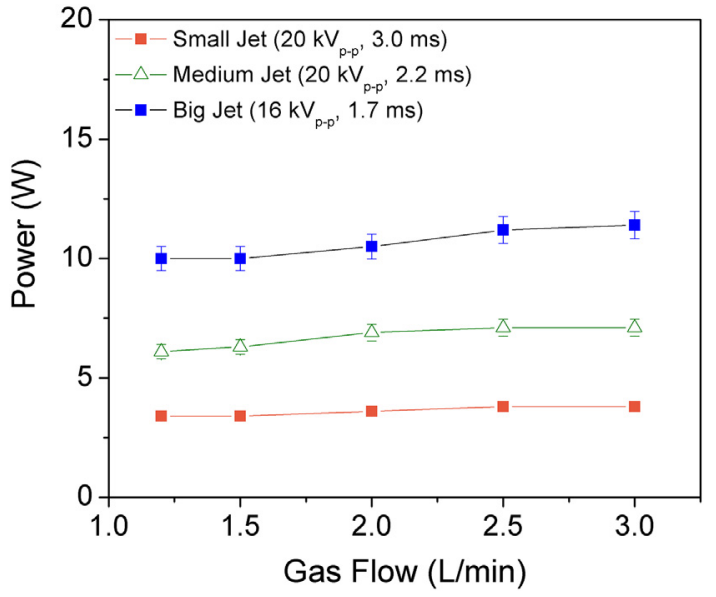

Fig. 6. Effect of the gas flow rate on the power for the three jets. Distance to the target was $1 \mathrm{~mm}$ for all jets.

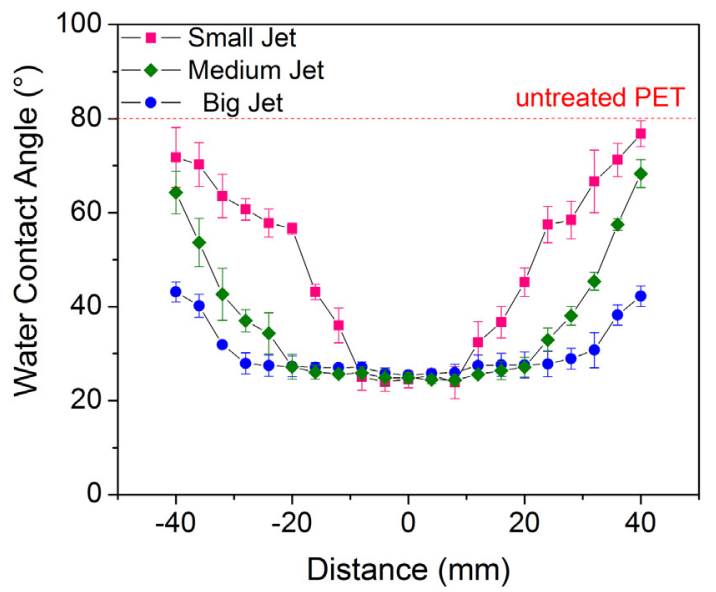

Fig. 7. Radial distribution of WCA for samples treated by the three different jets at the energy doses shown in the Table 1.

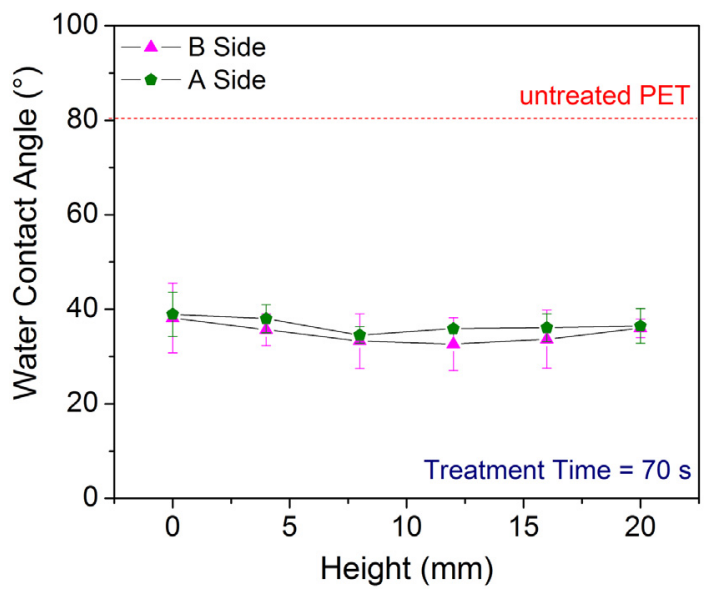

Fig. 8. WCA distribution of a PET sample placed vertically inside the big horn plasma jet. The energy dose was the same as the one in the Table 1 and the distance to the glass table was $1 \mathrm{~mm}$.

\subsection{XPS analysis}

The non-treated PET surface contained 75.9 at.\% of C and 21.9 at.\% of $\mathrm{O}$ as well as contaminations of $\mathrm{Cl}(\sim 2$ at.\%) and $\mathrm{Si} \mathrm{(in} \mathrm{traces).} \mathrm{After}$ all kind of plasma treatments the fraction of $\mathrm{O}$ in the central part of the
PET samples increased from 21.9 at.\% to $\sim 30$ at.\% while the fraction of $\mathrm{C}$ atoms decreased accordingly to $\sim 70$ at.\%. Similar results were obtained by some authors [33-36] using different plasma setups to surface treat PET.

Fig. 9(a) shows the elemental distribution along the sample S3 treated with the medium plasma jet. Both, the $\mathrm{O}$ and the $\mathrm{C}$ distributions exhibit plateau regions with length of about $50 \mathrm{~mm}$. Near the sample edges the elemental composition approaches the one of untreated PET sample. Also, a small insertion of about 1.0 at.\% of $\mathrm{N}$ was detected in the central part of the sample. Interestingly, the contaminations of $\mathrm{Cl}$ and $\mathrm{Si}$ in the central part of the PET samples were removed, leaving kind of footprint of the plasma jet with characteristic width (FWHF) $\sim 60 \mathrm{~mm}$ (see Fig. 9(b)). The elemental distribution of the sample S2 treated with the small plasma jet (not shown here) was very similar but with a slightly smaller ( $\sim 55 \mathrm{~mm}$ ) plasma jet footprint.

Fig. 10 depicts the $\mathrm{O}, \mathrm{C}, \mathrm{N}, \mathrm{Cl}$ and $\mathrm{Si}$ content along the sample S4 treated with the big plasma jet. As can be seen the $\mathrm{O}$ and $\mathrm{C}$ contents exhibit more or less uniform distributions with widths of about $75 \mathrm{~mm}$. Excluding the sample's extremities the $\mathrm{Cl}$ and $\mathrm{Si}$ impurities were removed by the plasma treatment, while $\mathrm{N}(\sim 1.0$ at.\%) was incorporated into the surface.

The shape of O content distributions along the samples S2, S3 and S4, treated with the small, the medium and the big plasma jets, respectively, is in a good agreement with WCA profiles presented in the Fig. 7. Similarly, the $O$ and $C$ distributions along the sample $S 5$ shown in Fig. 11 resemble the WCA measurements profile in the Fig. 8. The only exception is a bad point around $8 \mathrm{~mm}$ distance from the left sample edge. It is worth mentioning that XPS sampling diameter is only $250 \mu \mathrm{m}$ so this technique is much more sensitive to local sample inhomogeneity, which can be caused by some PET surface defects that may, in turn, also induce plasma treatment uniformity. On the other hand the WCA measurements, performed with $1.0 \mu \mathrm{l}$ droplets, are naturally averaged over the water drop size and thus are much less sensitive to local inhomogeneity.

The O/C ratio of the non-treated PET sample was $\sim 0.29$ and after the plasma treatment the $\mathrm{O} / \mathrm{C}$ ratios increased to $0.41-0.42$ for the big plasma jet and to 0.44 for the small and medium jets. According to authors [33] the increase in $\mathrm{O} / \mathrm{C}$ atomic ratio suggests that new oxygencontaining groups are formed on the PET substrate after the plasma treatment, which can explains the enhanced PET surface wettability observed in Figs. 7 and 8.

The bindings in high resolution $\mathrm{C} 1 \mathrm{~s}$ peaks were resolved into four components: aromatic $\mathrm{C}-\mathrm{C}$ bonds $(\mathrm{BE}=284.7 \pm 0.1 \mathrm{eV})$; $\mathrm{C}-\mathrm{O}$ bonds $(\mathrm{BE}=286.2 \pm 0.1 \mathrm{eV}) ; \mathrm{COO}$ bonding $(\mathrm{BE}=288.7 \pm 0.1 \mathrm{eV})$ and $\mathrm{a}$ shake-up at $\mathrm{BE}=291.2 \mathrm{eV}$. Fig. 12 depicts the $\mathrm{C}$ bindings of all PET samples. After all plasma treatments an increase of the amount of $\mathrm{O}-$ containing functionalities on the PET surface was detected.

\subsection{Roughness evaluation}

It is well known that reactive species generated by atmospheric pressure plasmas can greatly alter surface morphology and roughness of different polymers [29,37]. All plasma jet treatments in this study led to increase of PET roughness. Typical AFM images of the surface of pristine PET and samples treated with the small plasma jet and the big jet (horizontal treatment) are presented in Fig. 13 showing the 3D height map and morphology (deflection images). Images of the samples treated by the medium and the big jet (sample in vertical position) are not presented here because their surface morphology has very similar features as the one of the samples treated by the small jet and the big jet (horizontal sample), respectively.

The untreated sample exhibited a surface with numerous small peaks (see Fig. 13(a)). On the other hand, the small jet treatment in Fig. 13(b) resulted in formation of large globular structures on the polymer surface. Similar but smaller in size structures can be observed in the Fig. 13(c), where is shown the sample treated by the big plasma 

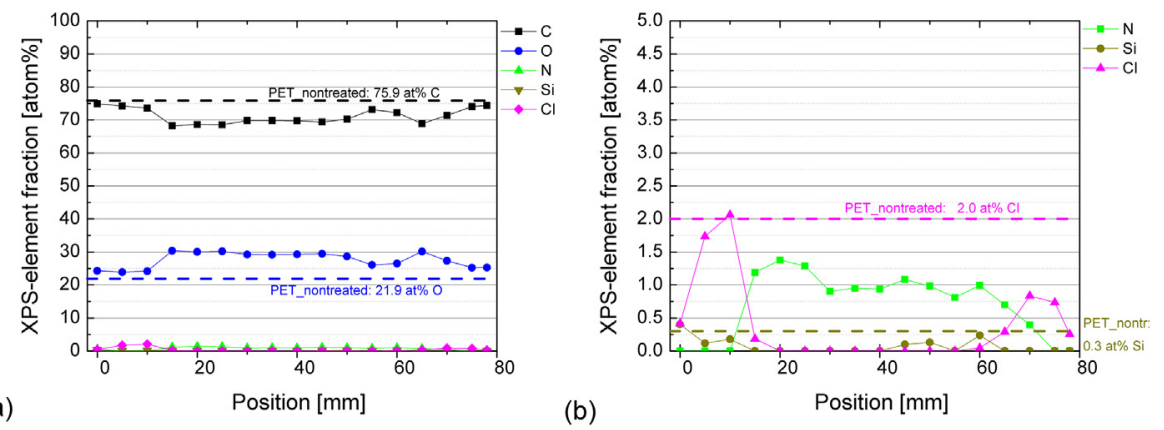

Fig. 9. (a). Elemental distribution along the sample $\mathrm{S} 3$ treated with medium plasma jet.

(b). Distribution of $\mathrm{N}, \mathrm{Cl}$ and $\mathrm{Si}$ for the medium plasma jet treatment.
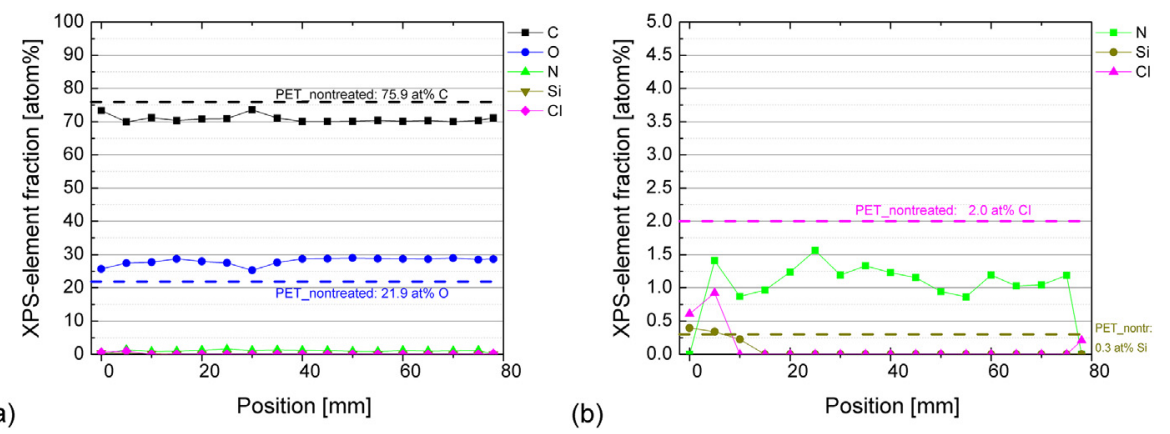

Fig. 10. Elemental distributions along the sample $\mathrm{S} 4$ treated with the big plasma jet: (a) O and $\mathrm{C}$ content and (b) Content of $\mathrm{N}, \mathrm{Cl}$ and $\mathrm{Si}$.

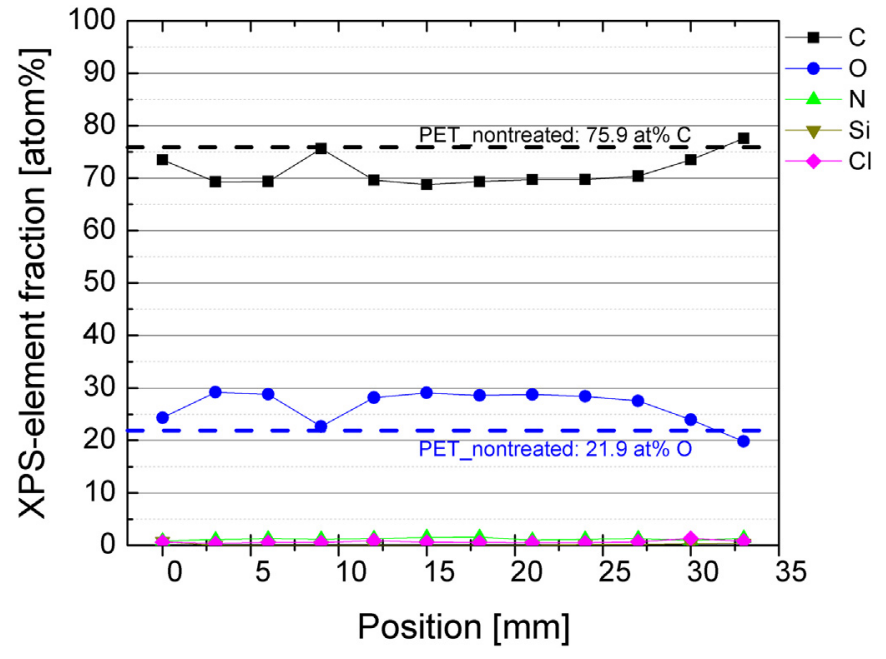

Fig. 11. $\mathrm{O}$ and $\mathrm{C}$ distributions along the sample S5 treated in vertical position with the big jet.

jet. These nodules can be explained by surface degradation and formation of highly oxidized short polymer fragments, also known as Low Molecular - Weight Oxidized Materials (LMWOMs) [37]. In a previous work [29] this behavior was also perceived for different kinds of polymers that were treated by plasma.

For each sample treated by plasma jet the polymer roughness was evaluated over two specific sample regions: one is on the area covered by the jet funnel and the second region is just outside the funnel edge. Then AFM analysis was performed over three arbitrary chosen spots $(2.0 \times 2.0 \mu \mathrm{m})$, the root mean square (RMS) roughness and deviation were calculated, into both region of interest. The roughness values are listed in Table 2.

Comparing the different plasma treatments it can be noticed a difference in the roughness values. The highest values of roughness (above

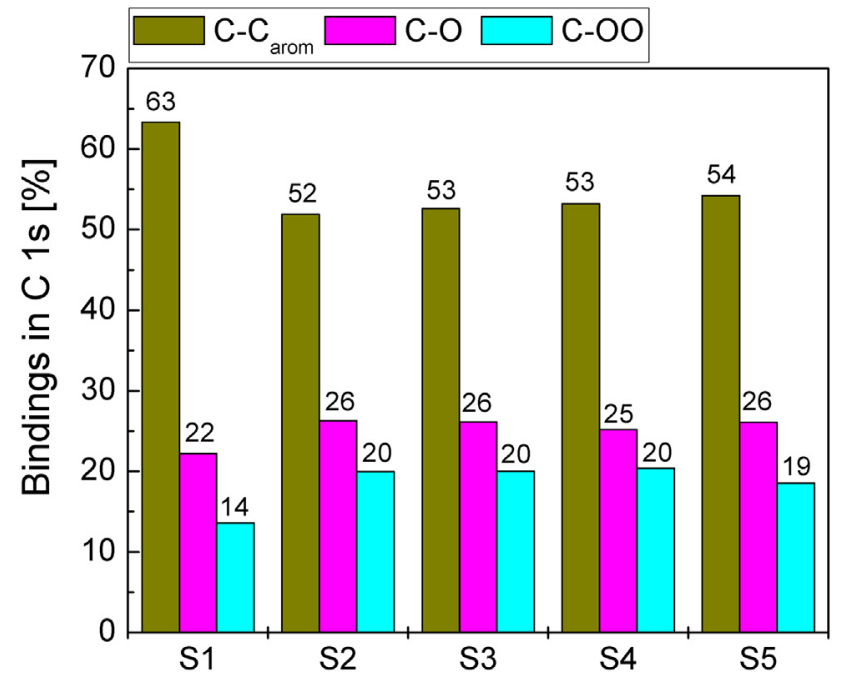

Fig. 12. Carbon bindings of the PET samples.

$6 \mathrm{~nm}$ ) were obtained for the samples treated by the small and medium jet, while the ones treated by the big jet (in horizontal and vertical position) exhibit roughness values around $1.5 \mathrm{~nm}$, which is only slightly above the roughness of the untreated sample $(\sim 1.1 \mathrm{~nm})$. Nevertheless this small roughness difference, the plasma effect on the polymer surface can be clearly seen in the Fig. 13 where surface morphology was changed substantially by the formation of small nodules. Polymer roughness outside the conical horn is always smaller than the roughness inside the funnel, but it is still higher compared to the untreated sample. The plasma effect extends outside the funnel, which is in a good agreement with WCA and XPS measurements. Also comparing the roughness values in the Table 2 with the data in the Table 1 and it can be concluded that the polymer roughness scales with the discharge power per unit area. However, based on the literature data [38-40] even though there was a change in the roughness values after plasma 

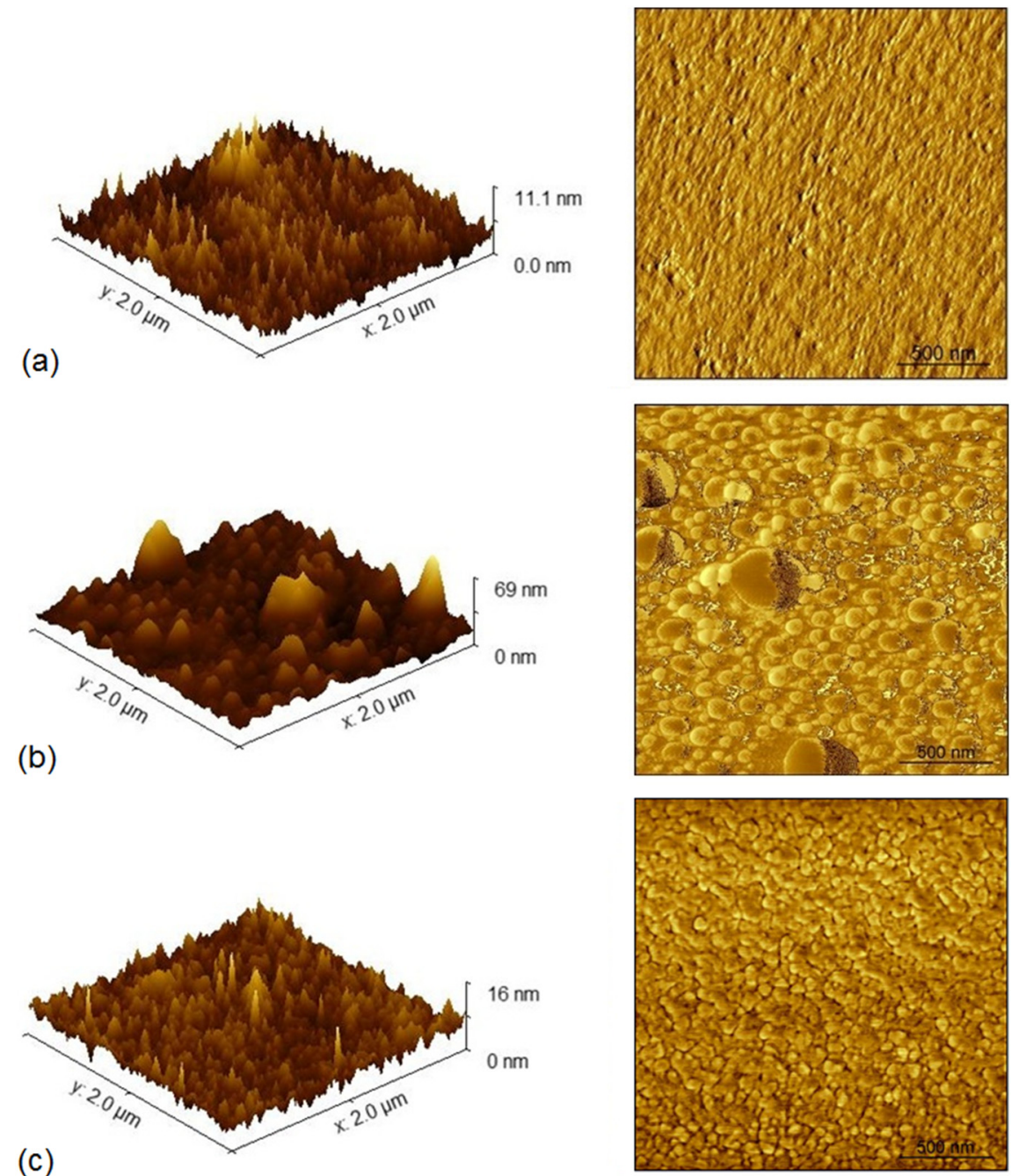

Fig. 13. AFM 3D height elevation and morphology (deflection images) of PET samples: (a) untreated; treated by (b) small jet and (c) big jet.

Table 2

Polymer roughness for each treatment condition.

\begin{tabular}{lll}
\hline Conditions & $\begin{array}{l}\text { RMS roughness inside } \\
(\mathrm{nm})\end{array}$ & $\begin{array}{l}\text { RMS roughness outside } \\
(\mathrm{nm})\end{array}$ \\
\hline Untreated & $1.15 \pm 0.26$ & $1.15 \pm 0.26$ \\
Small jet & $6.84 \pm 2.08$ & $4.05 \pm 0.95$ \\
Medium jet & $6.04 \pm 1.33$ & $3.78 \pm 0.76$ \\
Big jet & $1.46 \pm 0.23$ & $1.25 \pm 0.51$ \\
Big jet vertical sample & $1.46 \pm 0.20$ & - \\
\hline
\end{tabular}

treatment, the changes are less than a few nanometers. Therefore, it can be concluded that the roughness increase does not affect significantly the polymer wettability. In this case, the WCA reduction is mostly cause by surface functionalization and incorporation of polar species as detected by the XPS analysis.

\section{Conclusion}

Atmospheric pressure plasma jets are simple and inexpensive systems that are employed in a wide variety of applications including for surface modification of materials. However an inherited problem of these devices is the treatment uniformity especially for large area processing. In this work the effectiveness of atmospheric pressure plasma jet system with exchangeable exit nozzles for uniform surface modification of PET was demonstrated. Three different funnel exit diameters were employed in order to increase the area covered by the plasma. Incorporation of O-containing functional groups on the treated PET samples was confirmed by XPS analysis. Plasma treatments also resulted in an insertion of small amounts of $\mathrm{N}$ and also in removal of surface contamination of $\mathrm{Cl}$ and Si. The elemental distributions along the PET stripes exhibit plateau-like shapes whose characteristic widths scale with the horn size. Plasma processing at all conditions induced changes on the surface morphology and an increase of the polymer roughness, which scaled with the power per unit area of the treatment. The WCA reduction was mostly caused by surface functionalization and incorporation of polar species as detected by the XPS analysis, rather than the small changes in roughness values. Based on these results it can be inferred that the use of the horn-like nozzle was efficient in increasing the treatment area (up to $\sim 30 \mathrm{~cm}^{2}$ for the big plasma jet) and also in obtaining uniform surface modification with feasible operation conditions (low gas flow rate and $\mathrm{Ar}$ as plasma gas). It is also possible to treat small 3D samples with certain degree of uniformity. These results offer an insight about how changing the geometry of the plasma jet nozzle can significantly improve the treatment uniformity and increase the treated area of the PET polymer.

\section{Acknowledgements}

This work was supported by the Fundação de Amparo de Estado de São Paulo - FAPESP [grant number 2015/21989-6]. 


\section{References}

[1] P. Bruggeman, F. Iza, R. Brandenburg, Foundations of atmospheric pressure nonequilibrium plasmas, Plasma Sources Sci. Technol. 28 (2017) 455706.

[2] R. Brandenburg, Dielectric barrier discharges: progress on plasma sources and on the understanding of regimes and single filaments, Plasma Sources Sci. Technol. 26 (2017) 053001.

[3] J. Winter, R. Brandenburg, K.-D. Weltmann, Atmospheric pressure plasma jets: an overview of devices and new directions, Plasma Sources Sci. Technol. 24 (2015) 064001.

[4] A. Shashurin, M. Keidar, Experimental approaches for studying non-equilibrium atmospheric plasma jets experimental approaches for studying non-equilibrium atmospheric plasma jets, Phys. Plasmas 22 (2015) 122002.

[5] O.V. Penkov, M. Khadem, W.-S. Lim, D.-E. Kim, A review of recent applications of atmospheric pressure plasma jets for materials processing, J. Coat. Technol. Res. 12 (2015) 225-235.

[6] F. Fanelli, F. Fracassi, Atmospheric pressure non-equilibrium plasma jet technology: general features, specificities and applications in surface processing of materials, Surf. Coat. Technol. 322 (2017) 174-201.

[7] J. Winter, T.M.C. Nishime, S. Glitsch, H. Lühder, K.D. Weltmann, On the development of a deployable cold plasma endoscope, Contrib. Plasma Physics (2018) 1-11, https://doi.org/10.1002/ctpp.201700127.

[8] T. Abuzairi, M. Okada, S. Bhattacharjee, M. Nagatsu, Surface conductivity dependent dynamic behaviour of an ultrafine atmospheric pressure plasma jet for microscale surface processing, Appl. Surf. Sci. 390 (2016) 489-496.

[9] T. Wang, B. Yang, X. Chen, X. Wang, C. Yang, J. Liu, Distinct modes in the evolution of interaction between polymer film and atmospheric pressure plasma jet, Plasma Process. Polym. 14 (2017) 1-9.

[10] C.T. Liu, K.Y. Cheng, Z.H. Lin, C.J. Wu, J.Y. Wu, J.S. Wu, Effect of ground and floating electrode on a helium-based plasma jet and its applications in sterilization and ceramic surface treatment, IEEE Trans. Plasma Sci. 44 (2016) 3196-3200.

[11] Ö. Birer, Reactivity zones around an atmospheric pressure plasma jet, Appl. Surf. Sci. 354 (2015) 420-428.

[12] D. Shaw, A. West, J. Bredin, E. Wagenaars, Mechanisms behind surface modification of polypropylene film using an atmospheric-pressure plasma jet, Plasma Sources Sci. Technol. 25 (2016) 065018.

[13] A. Valinataj Omran, A. Baitukha, J. Pulpytel, F. Sohbatzadeh, F. Arefi-Khonsari, Atmospheric pressure surface modification and cross-linking of UHMWPE film and inside HDPE tube by transporting discharge, Plasma Process. Polym. 15 (2017) 1-12 e1700145.

[14] F. Rezaei, M.D. Dickey, M. Bourham, P.J. Hauser, Surface modification of PET film via a large area atmospheric pressure plasma: an optical analysis of the plasma and surface characterization of the polymer film, Surf. Coat. Technol. 309 (2017) 371-381.

[15] C. Zhang, M. Zhao, L. Wang, L. Qu, Y. Men, Surface modification of polyester fabrics by atmospheric-pressure air/He plasma for color strength and adhesion enhancement, Appl. Surf. Sci. 400 (2017) 304-311.

[16] I. Onyshchenko, N. De Geyter, R. Morent, Improvement of the plasma treatment effect on PET with a newly designed atmospheric pressure plasma jet, Plasma Process. Polym. 14 (2017) 1600200.

[17] Z. Cao, Q. Nie, D.L. Bayliss, J.L. Walsh, C.S. Ren, D.Z. Wang, M.G. Kong, Spatially extended atmospheric plasma arrays, Plasma Sources Sci. Technol. 19 (2010) 025003.

[18] Q.Q. Fan, M.Y. Qian, C.S. Ren, D. Wang, X. Wen, Discharge characteristics of a coldatmospheric-plasma jet array generated with single-electrode configuration, IEEE Trans. Plasma Sci. 40 (2012) 1724-1729.

[19] M. Ghasemi, P. Olszewski, J.W. Bradley, J.L. Walsh, Interaction of multiple plasma plumes in an atmospheric pressure plasma jet array, J. Phys. D. Appl. Phys. 46 (2013) 052001.

[20] F. Liu, B. Zhang, Z. Fang, M. Wan, H. Wan, K.K. Ostrikov, Jet-to-jet interactions in atmospheric-pressure plasma jet arrays for surface processing, Plasma Process. Polym. 15 (2017) 1-12 e1700114.

[21] N.O. Connor, H. Humphreys, S. Daniels, Plasma jet arrays, IEEE Trans. Plasma Sci 42 (2014) 756-758.

[22] H. Nizard, T. Gaudy, A. Toutant, J. Iacono, P. Descamps, P. Leempoel, F. Massines, Influence of discharge and jet flow coupling on atmospheric pressure plasma homogeneity, J. Phys. D. Appl. Phys. 48 (2015) 415204.

[23] H.M. Joh, H.R. Kang, T.H. Chung, S.J. Kim, Electrical and optical characterization of atmospheric-pressure helium plasma jets generated with a pin electrode: effects of the electrode material, ground ring electrode, and nozzle shape, IEEE Trans. Plasma Sci. 42 (2014) 3656-3667.

[24] H.R. Kang, T.H. Chung, H.M. Joh, S.J. Kim, Effects of dielectric tube shape and pinelectrode diameter on the plasma plume in atmospheric pressure helium plasma jets, IEEE Trans. Plasma Sci. 45 (2017) 691-697.

[25] S. Wang, J. Zhang, G. Li, D. Wang, Cold large-diameter plasma jet near atmospheric pressure produced via a triple electrode configuration, Vacuum 101 (2014) $317-320$.

[26] A. Yamamoto, Y. Kawano, M. Nakai, T. Nakagawa, T. Sakugawa, H. Hosseini, H. Akiyama, Investigation of gas flow dependence of plasma jet produced by pulsed power, IEEE Trans. Plasma Sci. 43 (2015) 3451-3455.

[27] B. Eliasson, U. Kogelschatz, Modeling and applications of silent discharge plasmas, IEEE Trans. Plasma Sci. 19 (1991) 309-323.

[28] K.G. Kostov, T.M.C. Nishime, M. Machida, A.C. Borges, V. Prysiazhnyi, C.Y. KogaIto, Study of cold atmospheric plasma jet at the end of flexible plastic tube for microbial decontamination, Plasma Process. Polym. 12 (2015) 1383-1391.

[29] K.G. Kostov, T.M.C. Nishime, A.H.R. Castro, A. Toth, L.R.O. Hein, Surface modification of polymeric materials by cold atmospheric plasma jet, Appl. Surf. Sci. 314 (2014) 367-375.

[30] K.D. Weltmann, E. Kindel, T. von Woedtke, M. Hähnel, M. Stieber, R. Brandenburg, Atmospheric-pressure plasma sources: prospective tools for plasma medicine, Pure Appl. Chem. 82 (2010) 1223-1237.

[31] A. Van Deynse, P. Cools, C. Leys, N. De Geyter, R. Morent, Surface activation of polyethylene with an argon atmospheric pressure plasma jet: influence of applied power and flow rate, Appl. Surf. Sci. 328 (2015) 269-278.

[32] I. Onyshchenko, A.Y. Nikiforov, N. De Geyter, R. Morent, Local analysis of pet surface functionalization by an atmospheric pressure plasma jet, Plasma Process. Polym. 12 (2015) 466-476.

[33] C. Borcia, I.L. Punga, G. Borcia, Surface properties and hydrophobic recovery of polymers treated by atmospheric-pressure plasma, Appl. Surf. Sci. 317 (2014) $103-110$.

[34] I. Junkar, A. Vesel, U. Cvelbar, M. Mozetic, S. Strnad, Influence of oxygen and nitrogen plasma treatment on polyethylene terephthalate (PET) polymers, Vaccum 84 (2010) 83-85.

[35] M. Gao, L. Sun, Y. Guo, J. Shi, J. Zhang, Modification of polyethylene terephthalate (PET) films surface with gradient roughness and homogenous surface chemistry by dielectric barrier discharge plasma, Chem. Phys. Lett. 689 (2017) 179-184.

[36] C. Huang, Y. Chang, S. Wu, Contact angle analysis of low-temperature cyclonic atmospheric pressure plasma modified polyethylene terephthalate, Thin Solid Films 518 (2010) 3575-3580.

[37] S. Guimond, M.R. Wertheimer, Surface degradation and hydrophobic recovery of polyolefins treated by air corona and nitrogen atmospheric pressure glow discharge, J. Appl. Polym. Sci. 94 (2004) 1291-1303.

[38] K.J. Kubiak, M.C.T. Wilson, T.J. Mathia, Carval wettability versus roughness of engineering surfaces, Wear 271 (2011) 523-528.

[39] T. Jacobs, N. De Geyter, R. Morent, S. Van Vlierberghe, P. Dubruel, C. Leys, Plasma modification of PET foils with different crystallinity, Surf. Coat. Technol. 205 (2011) S511-S515.

[40] A.V. Nastuta, G.B. Rusu, I. Topala, A.S. Chiper, G. Popa, Surface modifications of polymer induced atmospheric DBD plasma in different configurations, $\mathrm{J}$. Optoelectron. Adv. M 10 (2008) 2038-2042. 